\title{
Relazione educativa e comunicazione nella comunità di apprendimento
}

\author{
Anna Russo
}

\begin{abstract}
Il mutamento sociale in atto pone al centro dell'attenzione il "nodo" della formazione, fattore decisivo nell'odierna competizione globale. La riflessione sul lavoro scolastico, che sta alla base del processo di sviluppo professionale degli insegnanti del terzo millennio, deve comprendere la costruzione, con gli studenti, di un adeguato sistema relazionale, oltre che la competenza nel comunicare. Il valore delle relazione in ambito educativo non riguarda solo quella docente-studente, ma anche quella con i propri colleghi, oltre che con i genitori e, allargando lo sguardo, con l'intera comunità scolastica, definita non casualmente "comunità educante", nella quale le relazioni tra la scuola e la famiglia, la scuola e il mondo del lavoro e le altre agenzie educative vanno a creare una rete interdipendente di soggetti. Soggetti, questi, che condividono la responsabilità della crescita dei giovani, in un'ottica di inclusione e di ottemperamento ai diritti di uguaglianza e dignità, con lo scopo di ridurre le cause della povertà e del divario sociale, assicurando a tutti i il diritto allo studio e il successo formativo.
\end{abstract}

Parole chiave: scuola-famiglia, comunità educante, complessità, relazione educativa, comunicazione.

\begin{abstract}
The ongoing social change places the issue of training at the centre of development, which is a decisive factor in global competition. The debate concerning school-work, which is at the basis of the teachers' professional development process in the third millennium, must include the construction of an adequate relational system together with the students as well as a strong ability in communication. The value of relationships in the educational field does not only concern the student-teacher relationship, but also the one with colleagues, parents and the entire school community, therefore defined as the "educating community". In this kind of community, the relationships between the school and the family, the school, the "world of work", and other educational agencies therefore create a network of individuals, who share the responsibility for the growth of young people, with a view to inclusion and compliance with the rights of equality and dignity. They also aim at reducing the causes of poverty and the social gap to prevent abandonment and early school leaving, and to ensure the right to education and educational success to every student.
\end{abstract}

Keywords: school-family, educating community, complexity, educational relationship, communication.

${ }^{1}$ Dottoranda (curriculum del PhD: Mind, Gender $e$ Language) presso il Dipartimento di Studi Umanistici dell'Università degli Studi “Federico II" di Napoli. 


\section{La complessità dell'agire educativo}

In relazione allo sviluppo del soggetto, il mutamento sociale in atto induce a focalizzare l'attenzione sul tema della formazione, cruciale nella competizione globale. Nell'era della società della conoscenza, le istituzioni scolastiche si trovano al centro di un profondo ripensamento, sono chiamate a riconnettere i saperi formali a quelli non formali e informali, perché rivestono funzione importante nella crescita e nella maturazione umana e professionale degli studenti.

I più recenti documenti dell'UE indicano le strategie formative per rendere l'apprendimento attraente e per rinforzare i legami delle scuole con il mondo del lavoro, della ricerca e la società nel suo complesso. Difatti, il rapporto Eurydice Levels of Autonomy and Responsabilities of Teachers in Europe indica la difficile situazione della professionalità docente e la pressione verso la costruzione di un'offerta formativa più attraente per gli studenti, ciò imprime sulle traiettorie istituzionali uno specifico orientamento, con particolare ricaduta sulle scelte professionali degli insegnanti (Eurydice, 2008). I mutamenti sociali, i provvedimenti normativi, il riordino della struttura ministeriale, l'allocazione di importanti funzioni amministrative in materia scolastica presso gli enti locali, il riordino della formazione professionale e dell'apprendistato, sono tasselli di un mosaico teso a tratteggiare una nuova fisionomia dell'intero sistema educativo e formativo.

A livello nazionale, le innovazioni introdotte dal Titolo V della Costituzione e la conseguente trasformazione dell'assetto gerarchico del nostro Stato in un sistema a rete hanno portato alla trasformazione delle attribuzioni ai singoli soggetti (L. Cost. n. 3\2001)². Il processo di decentralizzazione ha avvicinato l'amministrazione ai cittadini, valorizzando le autonomie territoriali. Si è venuta così a configurare una nuova immagine di scuola incardinata nel territorio, capace di delineare una vera e propria politica scolastica, attraverso l'elaborazione di un progetto mirato, che va inquadrata nel contesto di quel processo di trasformazione e ammodernamento di tutta la P.A. che si è sviluppato in Italia nel corso degli anni Novanta del secolo scorso. In questa cornice, è venuta prospettandosi una scuola aperta, quale laboratorio permanente di ricerca, sperimentazione e innovazione didattica, di partecipazione e di educa-

${ }^{2}$ D'ora in poi, laddove non diversamente specificato, per i dettagli relativi alle emanazioni normative citate si rimanda ai Riferimenti normativi nazionali, N.d.R. 
zione alla cittadinanza attiva, garante del diritto allo studio, delle pari opportunità di successo formativo e di istruzione permanente dei cittadini, orientata alla massima flessibilità, diversificazione, efficienza ed efficacia del servizio scolastico, nonché all'integrazione e al miglior utilizzo delle risorse e delle strutture, all'introduzione di tecnologie innovative e al coordinamento con il contesto territoriale (L. n. 107/15). Una scuola, quella del ventunesimo secolo, che deve fronteggiare i cambiamenti in atto che riguardano le modalità di produzione, la trasmissione e diffusione della conoscenza, i modelli di apprendimento, le aspettative verso l'istruzione.

Il sistema scolastico è oggi di fronte alla necessità di ridefinire la propria strada; per farlo, è essenziale che chi la percorre lo faccia non solo con la responsabilità del guidare e del condurre, ma al contempo con l'idea di "servizio". Un percorso che non va tracciato autonomamente dai docenti, poiché, in una logica unilaterale e muscolare finirebbe per offuscare l'indispensabile funzione di leadership per l'apprendimento. La scuola è un'istituzione assai complessa che richiede una strategia di ascolto e condivisione della comunità scolastica con il territorio, con le famiglie, con enti di ricerca, Università e stakeholders.

Al fine di favorire il processo di miglioramento e innovazione della scuola italiana e dell'avvio di utili rapporti interistituzionali, si rende necessario il processo di ridefinizione delle componenti educative e formative chiave del territorio, quali scuola, famiglia, enti locali, associazionismo, strutture ricreative, mondo del lavoro e, anche, Aziende Sanitarie Locali. Nel 2012, l'allora Ministro dell'Istruzione dell'Università e della Ricerca Profumo aveva sottolineato l'esigenza che le scuole si configurassero come centri per la propria comunità e il territorio, facendo riferimento alla scuola come luogo fisico, inteso quale ambiente di interazione allargata e di confronto, che gradualmente superi gli spazi tradizionali dell'aula e dei corridoi e si configuri come luogo delle relazioni. Un luogo aperto, di coesione territoriale, sociale e di servizi alla comunità. Appunto, un vero e proprio «centro civico» (Profumo, 2012, passim).

John Dewey, nel lontano 1916, scriveva in relazione alla scuola che essa risulta un luogo privilegiato che assume la forma di vita sociale, una comunità in miniatura, in costante interazione con altre occasioni di esperienza associata (trad. it. 2004). Perché tutto ciò si concretizzi, occorre una piena presa di responsabilità di tutta la comunità territoriale; una partnership tra docenti e genitori, dalla quale scaturisca la valorizzazione del ruolo genitoriale, l'integrazione fra i diversi ruoli educativi ed infine la soddisfazione delle esigenze degli studenti. In una società carat- 
terizzata da ritmi di trasformazione rapidissima, l'idea di prefigurare il futuro degli studenti, e con essa la capacità di costruire dei percorsi di crescita, diventa più complessa per le agenzie educative.

Il mondo è diventato piccolo e ha assunto, di conseguenza, i comportamenti tipici di un «villaggio globale» (McLuhan, 1989, passim), dove le comunicazioni avvengono in tempo reale a grande distanza, con nuove frontiere di indagine che mirano non solo ai processi cognitivi individuali ma anche alle relazioni cognitive che l'individuo intrattiene con gli altri soggetti e ai processi di costruzione simbolica e di negoziazione in esse implicate. In un mondo pervaso da incertezze e difficoltà occorre favorire un inedito recupero dell'esperienza vissuta, che rifugge dalla ripetitività e dall'omologazione, che richiede come premessa l'educazione alla libertà ed esige la capacità di proiettarsi nel futuro custodendo il presente.

La trasformazione delle culture e delle identità, la complessità sociale e cognitiva, i nuovi modelli di intelligenza e di comunicazione diventano la sfida della complessità che, a sua volta, diventa anche sfida all'educazione e alla scuola. Complessità è, infatti, ciò che si scopre, insieme, semplice ed eterogeneo, variegato e unico allo stesso tempo. La scuola è immersa in una forte rete di interazioni che le richiedono, prestando ascolto alla lezione socratica, di costruirsi come comunità di pensiero che attraverso il dialogo condivide un percorso fatto di domande di senso, di significato, stabilendo con gli altri partecipanti un contatto positivo, volto alla collaborazione e alla messa in comune di idee e opinioni.

\section{La comunità educante}

Grazie all'impulso di una generale volontà della società civile di partecipazione alla vita pubblica, a partire dagli anni Settanta del secolo scorso, il sistema educativo ha contribuito alla dimensione inclusiva e accogliente del sistema sociale. In ragione di ciò, il sistema educativo di istruzione e formazione si connota di valori partecipativi che fanno riferimento agli obblighi di solidarietà e che promuovono il protagonismo attivo dei soggetti istituzionali (scuole autonome ed enti locali) e sociali (associazioni di genitori, associazionismo, mondo delle imprese ecc.).

L'idea è quella di una rete sociale, intesa come cittadinanza attiva, con la correlata capacità di inserirsi nei flussi delle decisioni per lo scambio e la condivisione di risorse professionali, istituzionali e informali. La condivisione degli obiettivi, il rispetto dei ruoli, le procedure di decisione e 
l'assunzione di responsabilità, nonché la qualità delle relazioni docenti/ genitori, docenti/docenti, docenti/studenti rappresentano fattori di qualità della scuola espresse nel D.P.R. n. 249/1998 con la previsione del Patto di corresponsabilità educativa, e anche dal D.P.R. n. 235/2007, con Lo statuto delle studentesse e degli studenti. Diventa prioritario l'obiettivo di favorire rapporti sempre più sinergici, basati sulla condivisione e la collaborazione. Epstein sottolinea come la scuola non sia composta da una diade, docenti e studenti, ma sia, o meglio debba essere, una learning community, composta da docenti, studenti, genitori e membri della comunità che cooperano per dinamizzare e arricchire l'istituzione scolastica e aumentare le opportunità di apprendimento e il benessere degli studenti (2009).

La scuola deve valorizzare particolarmente il contributo e la collaborazione dei genitori perché è il fattore più importante per migliorare l'apprendimento degli studenti. Un progetto canadese (Coleman, Collinge, Seifert 1993) mostra che l'atteggiamento positivo dei genitori verso la scuola influenza positivamente la motivazione e l'apprezzamento dei ragazzi nei confronti del proprio percorso di istruzione, giocando un ruolo fondamentale nel successo scolastico dei figli (PISA, 2003). Cresce il bisogno in ambito educativo di creare ponti tra scuola e famiglie, di favorire la partecipazione e la comunicazione e di rafforzare così la corresponsabilità nei confronti dell'educazione e della formazione degli alunni. La fondamentale dimensione relazionale del soggetto rende la comunicazione tra scuola e famiglia un processo determinante da attivare e costruire attraverso il parlare congiunto, lo scambio della parola fra pari (Boffo, 2011), l'ascolto dell'altro, il riconoscimento reciproco, e allo stesso tempo la cura, l'attenzione ma anche la possibile negoziazione, dei conflitti.

I genitori sono una risorsa molto importante per i figli e affinché possano rendersene conto e diventarne pienamente consapevoli devono essere considerati tre fattori chiave. Prima di tutto, essi devono ritenere significativo il loro ruolo con la consapevolezza dell'importanza del loro aiuto. In secondo luogo, devono sentire di avere un influsso, vedere che lo sforzo di aiutare porta il figlio a conseguire maggiori successi scolastici. Infine devono sentire sostegno, dalla comunità educante. I genitori che sentono di giocare un ruolo importante nel miglioramento del rendimento scolastico e nella partecipazione alla loro istruzione possono anche più facilmente arrivare a cambiare il proprio comportamento.

Tra la fine degli anni Settanta e i primi Ottanta, si afferma la visione ecologica di Urie Bronfenbrenner (1979, trad. it. 1986). La scuola e la 
famiglia fanno parte di un microsistema connesso con il mesosistema, cioè con l'insieme delle relazioni che intercorrono tra insegnanti e genitori. A loro volta, tali relazioni sono influenzate dall'esosistema, cioè da fattori esterni di varia natura ( $\mathrm{ad}$ esempio, l'occupazione dei genitori). Il tutto è inserito nel macrosistema, cioè nel contesto socio-culturale di riferimento.

Questa visione stimola la cooperazione, perché sottolinea la complementarietà di scuola e famiglia, incoraggiando la comunicazione e la collaborazione fra le due istituzioni. Bronfenbrenner introduce così, in una situazione multidimensionale dove ogni azione, ogni evento, ogni emozione è letta a livello macro, il contesto sovrastrutturale (leggi, politiche, valori, cultura); al meso ed eso troviamo le presenze istituzionali e gli organismi che da esse direttamente dipendono, interagendo con la sfera privata dell'individuo: direzioni istituzionali del lavoro, della giustizia, della città, insieme agli organismi lavorativi e sociali a cui il soggetto è connesso. Il micro, infine, esprime la rete delle relazioni dirette: famiglia, amici, vicini. L'elemento rilevante di tale modello è che, pur prendendo in considerazione il mondo esterno per delineare una teoria delle interconnessioni ambientali, ne delinea successivamente il loro impatto sulle forze che influiscono in modo diretto sulla crescita psicologica. Sono queste interconnessioni che possono essere decisive per lo sviluppo, quanto gli eventi che si danno all'interno di ogni situazione. L'individuo secondo il principio della dinamicità, non è considerato come una tabula rasa che l'ambiente modifica a suo piacimento, piuttosto, cresce e si muove in esso ristrutturandolo (Bronfenbrenner, 1979, trad. it. 1986). L'interazione tra individuo e ambiente è bidimensionale, l'uno modifica e influenza l'altro secondo un sistema di reciprocità. Le possibilità di crescita, di evoluzione e di benessere dell'uomo non dipendono da una causa singola ma sono legate a una complessa rete di strutture che comprendono gli individui con le loro specificità biologiche e psicologiche, l'ambiente, i gruppi, la società nel suo insieme.

Alle agenzie educative spetta un lavoro di rete che scaturisce dalla necessità di rifondare i grandi sistemi dello stato di benessere. Rifondare lo stato socio-assistenziale significa passare da una logica del chiedere ad una strategia del fare (sussidiarietà) ed anche la scuola dell'autonomia può essere interpretata con questo parametro (attraverso l'assunzione di responsabilità dal basso). I vari livelli della società hanno dunque il compito di sostenere le persone singole o associate. La scuola della sussidiarietà vive in una relazione di sviluppo e promozione con gli Enti locali e le altre agenzie presenti sul territorio, ed è essa stessa struttura sussidiaria per la sua utenza in un circolo sempre più virtuoso. Su tale 
sfondo il principio di sussidiarietà tempera e bilancia i nuovi rapporti che si instaurano tra gli artefici istituzionali dello stesso processo: famiglia, scuola, società, enti locali, istituzioni. Essi interagiscono in un partenariato virtuoso per promuovere e realizzare un servizio educativo a misura dell'alunno affinché possa raggiungere il successo formativo.

L'istituzione scolastica viene intesa come centro di formazione permanente attraverso un confronto democratico tra le forze che gestiscono il Paese. L'unicità del sistema educativo e formativo nei suoi diversi profili e aspetti strutturali e ordinamentali, richiede un confronto e un'interazione tra diversi soggetti e protagonisti. La scuola dell'autonomia è un universo di responsabilità dove tutto il personale è invitato, ognuno in proporzione alle proprie attitudini e predisposizioni, a una collaborazione continua per una scuola che risulti efficace ed efficiente. È impegno fondamentale di tutte le componenti della comunità scolastica le quali, nell'ambito degli specifici ruoli e responsabilità, concorrono ad assicurare il successo formativo delle bambine e dei bambini, delle alunne e degli alunni, delle studentesse e degli studenti (D.lgs. n. 66\2017).

Comunità è una parola calda, che evoca accoglienza, operosità collaborativa, partecipazione, collegialità, senso di appartenenza, valorizzazione delle persone, reciprocità, comunicazione: tutti fattori in grado di produrre ricchezza, oltre che "qualità sociale". Una comunità capace di costruire un canale permanente di ascolto come antidoto alla crisi gestionale, gioca un ruolo non solo strumentale e compensativo, ma strutturale e strategico. L'idea di comunità può quindi essere considerata come alternativa a visioni percepite come competitive, conflittuali, autoritarie o semplicemente fredde e burocratiche. La cura delle nuove generazioni è affidata a due istituzioni: la scuola e la famiglia. Ciascuna è guidata da una propria prospettiva nell'azione formativa portate all'ennesima potenza nella società postmoderna e multiculturale. Far diventare i genitori e gli altri soggetti della comunità membri attivi della learning community significa trasmette a tutta la comunità il messaggio che ciò che gli studenti imparano e quanto hanno imparato non è una questione che riguarda solo gli insegnanti e le altre persone che lavorano nella scuola, ma è una vera e propria priorità per tutto il contesto sociale circostante. Trotman sostiene che non soltanto i ragazzi hanno prestazioni scolastiche migliori e si comportano meglio quando i genitori sono coinvolti, ma anche i comportamenti dei docenti vengono influenzati positivamente dal coinvolgimento dei genitori (2001).

La riuscita dell'azione educativa trova le sue condizioni prioritarie in una affettuosa comunicazione interpersonale che in quanto tale richiede 
cura, rispetto, amorevolezza, benevolenza e fiducia. L'educazione, oltre che di tecniche e di strumenti, si alimenta di ideali e significati, di qualità relazionale e condotte affettive positive. L'impegno, l'energia e la motivazione dell'educando a trasformarsi è promosso e sostenuto in misura considerevole da un'interazione in cui egli si percepisce riconosciuto, stimato, importante, adeguato ed efficace (Mariani, 2006).

Di conseguenza, è assolutamente indispensabile da parte degli educatori costruire un'intersoggettività di relazioni, cui segua un percorso dinamico che si traduce in possibilità perennemente aperte a una molteplicità infinita di altre relazioni. La cura della dimensione relazionale e comunicativa nei contesti educativi promuove nello studente la nascita e il potenziamento di sentimenti di autostima ed empowerment piuttosto che di frustrazione o sfiducia. L' "intelligenza affettiva" dell'educatore risulta essere una capacità essenziale per una serie di trame relazionali che danno sostanza al lavoro educativo.

\section{La relazione educativa}

L'esperienza affettiva rappresenta una dimensione essenziale nel percorso di professionalizzazione dei docenti, ponendosi a fondamento della possibilità di tessere relazioni positive ed efficaci con i colleghi e con gli alunni, e di integrare la propria personalità e il proprio vissuto nella costruzione dell'identità professionale. L'identità professionale si basa su conoscenze disciplinari, capacità organizzative e progettuali e abilità operative che strutturano un bagaglio tecnico indispensabile, ma comunque insufficiente a tratteggiare la figura del bravo docente. Tale figura professionale va ampliata da uno sfondo formativo in grado di valorizzare gli aspetti emotivo-affettivi e comunicativo relazionali del fare scuola, prima e durante l'esperienza lavorativa. Si tratta di una formazione intenzionale e mai lasciata al caso, in grado di esprimere un contenimento emotivo e una meta-riflessione (Elia, 2017). Per tale ragione, investire sulla professionalità docente significa porre sempre maggiore attenzione al sapere riflessivo che emerge dall'interrogarsi continuo sui presupposti dell'azione professionale. Come evidenzia Clarizia (2017) si è, infatti, convinti che il personale livello di congruità e agio/incongruità e disagio (personale-professionale) del docente ne influenzi anche la competenza comunicativa. Uno spazio rilevante nella ricerca psicopedagogica, che in campo scolastico si interessa di fare luce sul cosiddetto curricolo implicito, ossia sulle dinamiche nascoste dell'esperienza di insegnamento-apprendimento. 
L'insegnamento come processo riflessivo richiede ai docenti di mettere in discussione le proprie strategie, metodologie e approcci teorici, oltre che i pregiudizi professionali, le convinzioni e i punti di vista, di riesaminare costantemente obiettivi, pratiche e saperi, sia individualmente che all'interno della comunità professionale (Fabbri, Striano, Melacarne, 2008), acquisendo un habitus permanente che consente l'auto-regolazione e l'apprendimento della professionalità. In tali contesti, dunque, la dimensione riflessiva come funzione regolativa dell'azione svolge un ruolo di primo piano, garantendo all'educatore di sapere cosa fa quando agisce (Striano, 2001). L'educatore in quanto professionista è portatore di conoscenze e di competenze che prendono forma anche attraverso un confronto quotidiano con la realtà educativa che richiede un interrogarsi sul proprio agire professionale, a partire da pratiche riflessive sull'azione e attraverso l'azione: si è trattato quindi di una formazione in situazione, basata sulla circolarità tra esperienza, riflessività e professionalità (Mortari, 2009).

La disposizione a valorizzare il rapporto interpersonale tra educatore e educando come un rapporto schietto e umano è rintracciabile sin nelle società arcaiche. La socialità umana si configura nella relazione educativa con una duplice caratterizzazione: circolare e asimmetrica, dove l'educatore si pone a servizio della graduale conquista della maturità dell'educando. Ogni intervento richiede una tensione verso l'agire accompagnata dallo studio di come renderla efficace rispetto allo scopo. Ciò pone l'educatore in uno stato di responsabilità, di ricerca e azione costante, di negoziazione di significati, di tessitura di rapporti, di riflessione. Nella sua accezione positiva l'autorità è tale se permette e salvaguarda la libertà dell'educando in vista del successo formativo e della piena realizzazione. L'educando va introdotto nella pratica della disciplina perché, solo attraverso il tirocinio dei limiti posti dall'autorità dell'educatore, diventa capace di imporseli da sé conquistando la maturità (Mari, 2009). La libertà, quale nota essenziale della persona, segna il limite dell'autorità e lo specifico dell'educazione l'idea di libertà come dimensione mai compiuta ma componente integrante di ogni processo educativo. Il fine dell'educazione è rendere capace l'educando di praticare la libertà come maturità. L'educando è quindi protagonista dell'azione educativa e portatore di saperi e risorse entro un ecosistema con il quale vi è una relazione caratterizzata da interdipendenza.

Per quanto le concrete e quotidiane relazioni possano, non raramente, presentarsi come imperfette, accidentate o attraversate da inevitabili dolorosi tradimenti (Turnatutri, 2000), lo spazio della relazione, almeno 
in quei luoghi comunicativi interpersonali che il discorso pedagogico continua a ipotizzare, si può connotare, definire e descrivere come educativo e/o formativo per le generazioni future.

La riflessione sul lavoro scolastico, che sta alla base del processo di sviluppo professionale degli insegnanti, del terzo millennio, deve comprendere la costruzione, con gli studenti, di un adeguato sistema relazionale. Un'esperienza di apprendimento richiama fattori quali l'efficacia dell'insegnamento, le relazioni positive con l'insegnante e il gruppo, l'importanza del bisogno di autostima e di appartenenza, la soddisfazione nell'eseguire un'attività. Gli aspetti cognitivi si intrecciano con quelli socio affettivi degli individui con le relazioni dinamiche che questi individui intrattengono con il contesto in cui operano. Il paradigma scolastico sta cambiando e con esso la domanda educativa, che si è fatta più complessa e non si accontenta più dei contenuti, ma presta attenzione anche alla forma del comunicare, diventa importante stabilire una relazione, cioè un rapporto umanamente soddisfacente. È fondamentale che gli insegnanti coltivino rapporti di comunicazione personale e relazione educativa con i propri alunni; questo fra l'altro consente loro di cogliere con prudenza segnali espliciti o impliciti di condizioni di disagio o sofferenza personale o familiare. La vicenda emotiva inevitabilmente connessa all'apprendimento e alla relazione educativa richiede una gestione non sempre facile e alquanto faticosa da parte degli adulti di riferimento.

Le ricerche scientifiche nel campo delle neuroscienze sembrano confermare ciò che già Vygotskij (1930, trad. it. 1987) aveva intuito, cioè che le esperienze hanno una grande influenza nello sviluppo cerebrale e quindi nella creazione e selezione di connessioni sinaptiche, grazie alla plasticità neurale (Rizzolatti, Sinigaglia, 2006). Lo sviluppo del cervello non è dato solo da fattori biologici e genetici, ma anche da quelli ambientali: lo sviluppo è in questo senso «esperienza-dipendente» (Giorgi, Lo Verso, 2008, p. 285).

In tale prospettiva si assegna di fatto un ruolo all'adulto come facilitatore e mediatore delle relazioni. Dal suo canto, il docente nel contesto scolastico non si rapporta ad un singolo allievo ma ad un intero gruppo classe, composto da differenti individualità. È posto inoltre davanti a problematiche non solo a carattere prettamente educativo, ma anche psicologico, legate alla crescita così come a possibili e sempre più frequenti aspetti di disagio emozionale. Gli studenti, come individui e come gruppo, portano con sé una varietà di obiettivi strettamente legati ai loro bisogni, sono naturalmente portati verso il soddisfacimento di obiettivi di tipo cognitivo (ad esempio acquisire competenze, aumen- 
tare la propria autoefficacia, soddisfare i propri interessi intellettuali) e si associano a obiettivi di tipo affettivo ( sentirsi a proprio agio) e sociale (essere accettati in un gruppo essere riconosciuti come individuare e ottenere sostegno, esercitare un senso di superficialità) (De Beni, Moé, 2000).

L'educatore, nel suo agire come tale, fa al contempo esperienza del proprio limite e della solidarietà con l'altro, rendendosi conto non solo di ciò di cui ha bisogno il particolare educando, ma anche di ciò che egli stesso è in grado di offrire alla persona che ha di fronte. La relazione educativa si compie come relazione di aiuto (Rogers, 1957, trad. it. 1970), cioè come un rapporto in cui una persona si attiva per facilitare la crescita e la maturità dell'altro. In un rapporto di reciprocità lo studente non si configura come soggetto da manipolare, ma come persona capace di autocompimento e di autorealizzazione,

Carl Rogers pensa all'uomo come a un fascio di potenzialità positive che all'educazione spetta sviluppare e alla terapia ripristinare nel caso qualche elemento negativo abbia interrotto la loro attualizzazione. Il compito di chi si fa cura dell'altro (il cosiddetto «professionista delle relazioni» (Ibidem, passim): genitore, insegnante, educatore, terapista, ecc.) è perciò quello di rafforzare gli aspetti positivi costitutivi l'individuo. Questo è possibile solo quando le relazioni interpersonali si svolgono senza la pretesa dell'essere forte di decidere per l'essere debole, ma nella logica della relazione d'aiuto, che costituisce uno dei concetti chiave nella teoria rogersiana. La relazione d'aiuto si compie ogni qualvolta si cerca di favorire nell'altro la crescita, lo sviluppo, la maturità, un miglior funzionamento e una più salda capacità di affrontare la vita.

Per essere efficace, la relazione ha bisogno di svolgersi in un clima di accettazione incondizionata dell'altro (senza la pretesa, per esempio, di giudicarlo o di orientarlo direttiva mente), di relazione empatica (mediata cui si verifica la reciproca condivisione), di autenticità e di congruenza e cioè senza maschere, facciate, doppia verità e cioè, in una parola, in un contesto non direttivo. La fiducia nel potenziale dell'altro, in particolare, rappresenta la base fondamentale per qualsiasi relazione interpersonale costruttiva in campo sia terapeutico sia educativo.

La relazione di aiuto poggia dunque su tre condizioni fondamentali: la congruenza, l'accettazione positiva incondizionata e l'empatia. L'educatore può sostenete la consapevolezza negli studenti dei propri sentimenti e vissuti, promuovendo il riconoscimento della persona umana come unica e originale, nella sua totalità, con difetti e qualità, senza critiche o valutazioni. Nella prassi educativa bisogna, allora, avviare 
una comprensione autentica che si esplica nella capacità di mettersi nei panni dell'altro, di coglierne con sincerità e rispetto il mondo interiore, contenuti emozionali e cognitivi. Sicuramente il motivo della dimensione emotiva e delle emozioni nell'esperienza educativa e di insegnamento-apprendimento rappresenta una nuova emergenza educativa dato il nesso tra mondo interiore, vissuti, affetti e educazione.

L'allievo acquista responsabilità se è inserito in un contesto educativo favorevole, in cui i bisogni vengono soddisfatti. Il docente attraverso una gestione del gruppo classe che privilegia un approccio educativo capace di coinvolge gli studenti, li sollecita ad esprimersi, propone attività personalizzate, insiste sull'autovalutazione, favorisce la qualità delle relazioni, usa metodi persuasivi e non repressivi. La costruzione di un ambiente di apprendimento si fonda sulla qualità delle relazioni interpersonali e sulla creazione di un clima sociale positivo, caratterizzato da accoglienza, sicurezza, fiducia, autonomia, autostima, aiuto e collaborazione. L'OMS, nel 1993, nel report intitolato Life skills education in school, in merito all'individuazione e alla definizione delle abilità fondamentali personali e relazionali che servono per governare i rapporti con il resto del mondo e per affrontare positivamente la vita quotidiana fa un importante riferimento alla dimensione emotiva e corporea di tali abilità (Ibidem). L'importanza dell'educazione, e nei diversi contesti/opportunità, che rendono possibile lo sviluppo di questa dimensione. Si tratta di aiutare l'individuo a maturare la competenza emotiva e le abilità emozionali, che secondo Goleman, comprendono l'autoconsapevolezza, la capacità di identificare, esprimere e controllare i sentimenti, frenare gli impulsi e rimandare la gratificazione, controllare la tensione e l'ansia (1995, trad. it. 1997). Diventano pertanto importanti fattori per il docente: il controllo prossimale (la vicinanza del docente blocca la volontà di infrangere le regole); il contatto oculare (per esprimere direttive e scoraggiare condotte non idonee); la postura (per sottolineare l'importanza di un momento didattico e dissuadere comportamenti inadeguati); la gestualità (per rinforzare le direttive, trasmettere agli alunni difficili la propria calma e il controllo della situazione); il tono della voce (per comunicare i propri stati d'animo).

Entrare in contatto con il mondo affettivo degli alunni (sia nell'atto di apprendere sia quando vogliamo dare loro aiuto, o quando li ascoltiamo), ci permette di incontrare e capire le loro emozioni e gli stati d'animo, i sentimenti e gli atteggiamenti. Nelle Indicazioni Nazionali per il primo ciclo di studi del 2012 viene sottolineato quanto la scuola, intesa come comunità educante, debba diffondere la cosiddetta convivialità 
relazionale. Si tratta di intessere la vita scolastica di linguaggi affettivi ed emotivi in grado di promuovere le condivisioni di quei valori che fanno sentire i membri della società come parte di una comunità vera e propria. L'intelligenza emotiva è considerata da Salovey e Mayer come la capacità di monitorare e dominare le emozioni proprie e altrui e usarle per guidare il pensiero e l'azione. Goleman riprende questo concetto definendo l'intelligenza emotiva la capacità di motivare se stessi, di perseguire un obiettivo nonostante le frustrazioni, controllare gli impulsi e modulare i propri stati d'animo, evitando che le sofferenze ci impediscano di pensare. La competenza emotiva si esplica attraverso elementi quali: la consapevolezza del sé, l'autocontrollo, la motivazione, l'empatia e l'abilità sociale. Gli studenti che hanno maggiori competenze interpersonali ed intrapersonali mostrano capacità di adattamento e gestione dello stress in situazioni difficili. Il processo alterocentrico rappresenta il presupposto per la negoziazione dei reciproci punti di vista e, di conseguenza, per la prevenzione dei conflitti. La professione docente non è fatta solo di competenze disciplinari, ma si basa anche sulla capacità di gestire efficacemente la comunicazione con gli alunni.

La cura e la promozione del clima di classe dipende dall'intrecciarsi di molti fattori tra loro continuamente interagenti e, in particolare, dal modo in cui vengono gestite variabili complesse come: il potere, l'autorità e la leadership, l'equilibrio tra bisogno di struttura e bisogno di autonomia negli studenti, la formulazione di norme di comportamento, dei criteri di comportamento e dei criteri di valutazione, l'uso di modalità di lavoro cooperative e la quantità, la qualità e i formati della comunicazione.

Per "clima di classe" s'intende la percezione soggettiva che gli studenti hanno dei docenti, delle discipline e dei compiti di apprendimento. L'impegno ad apprendere, l'entusiasmo per il compito da svolgere, l'attivazione delle risorse personali sono destati e sostenuti non poco da un clima relazionale in cui il soggetto in formazione si percepisce riconosciuto, amato, stimato, importante, di valore, adeguato, efficace. In virtù di una buona gestione delle dinamiche e degli eventi affettivi. Vivere una relazione con i compagni caratterizzata da un clima di conoscenza e fiducia reciproca, rappresenta uno dei fattori fondamentali nell'acquisizione delle competenze; sperimentare un clima di classe positivo significa percepire un ambiente in cui le proprie opinioni e i propri interessi vengono presi sul serio e nel quale la propria partecipazione viene accolta come risorsa e come valore per tutta la comunità-classe (Bruner, 1986, trad. it. 1988). 
Nel processo educativo e didattico è importante promuovere la creazione di efficaci ambienti di apprendimento, in cui si alimentano rapporti personali con ciascun allievo, una condotta equa e leale nei confronti di tutto il gruppo di alunni, attraverso una comunicazione interpersonale docente discente. La comunicazione interpersonale assume, nel contesto educativo, una rilevanza fondamentale per sostenere le attività. Esiste una stretta relazione tra una "comunicazione positiva" e i processi di apprendimento.

Il docente ha il compito della gestione della classe e delle relazioni, che si rivela uno dei più delicati e complessi. Rogers definisce la figura dell'insegnante «facilitatore della comunicazione» (1957, trad. it. 1970, passim). Il docente è chiamato a curare il livello relazionale e comunicativo, a promuovere relazioni pro-sociali, anche fra gli allievi stessi. 亡̀ dunque essenziale una comunicazione efficace evitando atteggiamenti aggressivi, sanzionatori e polemici.

\section{Conclusioni}

Il valore della relazione in ambito educativo, non riguarda solo quello docente studente ma anche quello con i colleghi, con i genitori, con l'intera comunità scolastica. Un percorso dinamico che si traduce in possibilità perennemente aperte ad una molteplicità infinita di altre relazioni. La complessità intrinseca all'atto educativo, il ruolo svolto dagli habitus di ciascuno degli attori coinvolti, la mancata formazione di questi ultimi alla collaborazione, i frame socio-culturali in cui l'incontro si svolge, rendono le relazioni faticose.

Il ruolo dei genitori nella vita scolastica risulta sempre più determinante, trasformandosi da mera partecipazione ad autentica cooperazione alla progettualità e ai processi formativi. Il rapporto scuola famiglia evolve nel tempo verso una relazione sempre più interattiva. L'obbligo per le istituzioni scolastiche, in linea con le disposizioni normative è di investire la famiglia di corresponsabilità educativa. L'oggetto del rapporto famiglia scuola, la formazione delle giovani generazioni, convoca gli adulti di una comunità alla condivisione della responsabilità (D.P.R. n. 235/2007; Nota Ministeriale n. 3602/2008). La progettualità educativa diventa il fulcro della corresponsabilità scuola famiglia, che si traduce in un agire di concerto, nel rispetto di un principio pedagogico basilare: la coerenza. Le dichiarazioni di principio desunte dal quadro normativo, trovano con difficoltà il pieno compimento. 
L'incontro tra scuola e famiglia si regge su un anello fragile, pronto a spezzarsi al primo ostacolo. La scuola, nell'attuale scenario, entra in contatto continuo con una controversa realtà familiare che appare frammentata, stratificata, di un nucleo fondante, non sempre capace di offrire il senso di una comunità e in grado di supportare e di rappresentare ancora una differenza generazionale (Recalcati, 2011).

L'incontro tra scuola e famiglia ha luogo all'insegna di una molteplicità di prospettive. La letteratura internazionale e diverse ricerche empiriche (Dusi, 2012) condotte nell'ambito delle scienze umane, richiedono a queste due agenzie educative di tessere proficue alleanze educative e una partnership efficace al fine di influire positivamente sulle performance degli studenti nel rispetto delle reciproche competenze. Il processo di costruzione dell'alleanza educativa tra la scuola e la famiglia non può prescindere dalla parallela tessitura di proficue relazioni interistituzionali. Nell'ambito della relazione scuola famiglia prendono forma le mediazioni possibili tra il formale \istituzionale e l'informale.

Le istituzioni scolastiche sono chiamate a promuovere in tal senso attività di informazione e di comunicazione, funzione essenziale per garantire un sistema di comunicazione organizzativa adeguato sia interno, sia esterno, per la comunità educante (docenti, personale ATA, genitori, alunni, stakeholders). A partire dagli anni Novanta, infatti, in corrispondenza con il processo di decentramento amministrativo, la spinta alla comunicazione della pubblica amministrazione si è fatta sempre più forte: il processo ha avuto inizio con la L. 241/1990 per concludersi con la L. n. 150/2000. Quest'ultima, in particolare, insieme alla Direttiva Ministeriale che ne ha chiarito l'applicazione, definisce le attività di informazione e comunicazione istituzionale proprie delle P.A. e stabilisce che devono essere volte a conseguire l'informazione (è la funzione che svolge il sito della scuola); comunicazione esterna rivolta ai cittadini (in questo caso alla rete di stakeholders della scuola e alle famiglie) e comunicazione interna.

Nella scuola la comunicazione è strumento essenziale per la gestione dei processi di sviluppo dell'autonomia, della qualità dei risultati e delle risorse umane nella realizzazione di una partecipazione più ampia al progetto educativo di Istituto. La comunicazione organizzativa, (Invernizzi, 2000), in tal senso, può rappresentare uno strumento operativo per la creazione di scambio e di condivisione di messaggi informativi e valoriali all'interno delle diverse reti di relazioni (docenti/docenti, docenti〉 genitori, docenti\alunni) che costituiscono l'essenza dell'organizzazione e della sua collocazione nell'ambiente. La comunicazione, sia interna sia 
esterna, insieme all'informazione e alla condivisione, stimola l'identificazione e il senso appartenenza. Diffondere una cultura comunicativa può avvicinare le famiglie e gli studenti alla scuola per farli evolvere e crescere insieme, per condividere un'unica missione.

Lo scambio comunicativo non è un semplice elenco delle attività svolte dalla scuola. Piuttosto, attraverso la rendicontazione, la scuola valuta con approccio critico e comunica in modo trasparente con approccio fiduciario il ritorno educativo che essa è stata capace di assicurare alla comunità utilizzando le risorse di cui dispone. In questo modo vengono coinvolti tutti gli stakeholders (non solo le famiglie) nella comprensione dei bisogni educativi e formativi a cui la scuola deve fare fronte nella sua azione. Le Linee di indirizzo lo considerano uno strumento fortemente aggregante, attraverso il quale le famiglie, coinvolte sin dall'inizio in questo processo e individuate quali interlocutori privilegiati, potranno esercitare il loro ruolo propositivo realizzando una progettazione partecipata.

L'atmosfera si pone come vero e proprio medium educativo. Spazio, tempo e linguaggio sono le forme che mediano la relazione con il mondo. L'atmosfera è la condizione educativa che consente l'apertura alla comprensione dell'estraneo e dell'improprio ed essa è pedagogicamente promossa mediante quella tonalità emotiva ineludibile che per Bollnow è la fiducia (1956, trad. it. 2008). La complessità del sistema comunicativo scolastico richiede agli insegnanti flessibilità e capacità di riconoscere le variabili che costituiscono la situazione comunicativa di un evento: contesto, argomento, ruolo dei partecipanti, scopi dichiarati e non, atteggiamenti psicologici, uso adeguato di tutte le funzioni della comunicazione.

Parallelamente, le attuali normative ministeriali richiedono il rispetto dei tempi di insegnamento, al fine di garantire l'appropriatezza del programma. Ciò tuttavia potrebbe incidere sulla qualità della relazione educativa. In questa ultima, il docente che utilizza opportune modalità comunicative verbali e non verbali, come incoraggiare, sollecitare, accompagnare, può favorire nei propri studenti una migliore maturazione di diverse competenze, che si esprime non solo nello sviluppo delle conoscenze e delle abilità, ma anche nello sviluppo di abilità relazionali e vissuti emozionali positivi nell'apprendimento che contrastino l'impotenza appresa, (Seligman, 1990, trad.it 1996) ovvero quella particolare percezione delle proprie abilità quali insufficienti o inefficaci nel risolvere una situazione problematica o un compito, che il soggetto può sviluppare a seguito di ripetuti fallimenti o nel contesto di una scarsa autostima 
(Bandura, 1996, trad. it. 2000), che in alcuni casi può diventare causa di abbandono o rifiuto della scuola. Il vissuto emozionale negativo in contesti di apprendimento può creare vissuti di disagio, frustrazione o di blocco, piuttosto presenti tra gli studenti delle scuole, fino a casi di vera e propria fobia scolare.

La principale potenzialità dell'essere umano, compresi bambini anche molto piccoli, si manifesta nell'attitudine a comunicare, a entrare in rapporti simbolici con gli altri. Nessuno può costruire la propria esperienza e il proprio sapere da solo e va dunque posto nelle condizioni di farlo: questo è possibile solo se «qualcuno comunica qualcosa». Il soggetto che cresce ha bisogno di relazioni che attivino la sua coscienza, che lo introducano nel mondo e lo aiutino a farne un'esperienza sensata, il ricorso della parola e del dialogo per una relazione cosiddetta generativa (Ducci, 1983). La relazione «tra uomo-e-uomo» è posta da Martin Buber in termini di salvezza dell'uomo contemporaneo ormai senza casa, e cioè senza un'immagine stabile del mondo. Solo nella relazione con l'altro l'uomo ritrova se stesso e supera la solitudine e l'isolamento. Un altro fondamentale elemento della relazione umana diviene il Noi, che comprende l'Io e il Tu, e si realizza soltanto quando si consuma appunto la relazione «Io-Tu» (1923, trad. it. 1993, passim). L'incontro costituisce l'occasione educativa per eccellenza per uscire da sé e vincere la tentazione dell'individualismo egocentrico, condizione fondamentale perché questo avvenga è l'esercizio della libertà da parte di entrambi i protagonisti dell'incontro (Romano Guardini, 1987). Il male dell'uomo consiste nella solitarietà, nella mancanza del "tu". La malattia dell'uomo è riducibile all'essere chiuso al tu e la parola umana ha la funzione propria del risanamento della malattia/chiusura dell'Io (Ebner, 1921, trad. it. 1998).

Sarebbe opportuno, piuttosto che affidarsi alle prassi consuete (" $a b$ biamo sempre fatto cosi..."), avviare l'elaborazione di un vero e proprio piano di comunicazione interna: in tal modo si avrebbe un passaggio rilevante da una comunicazione interna a una comunicazione organizzativa. Il piano dovrebbe contenere la definizione di obiettivi, la scelta degli strumenti di attuazione, la previsione dei tempi di realizzazione, monitoraggio e revisione del piano stesso. In termini esemplificativi è possibile pensare al macro obiettivo, previsto per altro dall' art. 13 del D.P.R. n. 62/2013, di favorire l'instaurarsi di rapporti cordiali e rispettosi tra i collaboratori, che impegna le istituzioni scolastiche ad assumere iniziative finalizzate alla circolazione delle informazioni, alla formazione e all'aggiornamento del personale, all'inclusione e alla valorizzazione delle differenze di genere, di età e di condizioni personali; per quanto riguar- 
da gli strumenti è oggi semplice e a costi contenuti la possibilità di creazione di caselle di posta personalizzate, attraverso le quali far circolare le informazioni in maniera diffusa (facendo attenzione a non esagerare: in tal senso in contrattazione sarà discusso il diritto alla disconnessione).

La comunicazione viene studiata anche dal ruolo che ricopre nella vita interna delle organizzazioni scolastiche. In questo senso la comunicazione è un elemento necessario per la gestione delle relazioni sociali e la regolazione dei conflitti. La comunicazione interna negli anni della qualità totale dell'efficacia e dell'efficienza del servizio si è ritagliata un ruolo di collante culturale, di veicolo di identità comune, di orientamento univoco, di condivisione di missione, di visioni e strategie. La comunicazione è legata al concetto di fiducia e di reputazione. La comunicazione (comportamenti, segni, relazioni) si configura come un decisivo strumento per conferire stabilità ed efficacia al sistema educativo, un'area della professionalità su cui intensificare gli sforzi di ricerca e formazione.

La capacità di ascoltare e ascoltarsi insieme alla capacità di osservare il proprio contesto avvia un confronto autentico. La riflessione allora porta a ripensare le pratiche professionali affidando un maggiore spazio a quelle che sono le interazioni con i colleghi promuovendo quelle pratiche di instructional leadership e knowledge transfer. Una leadership distribuita (Paletta, 2013) capace di generare una cultura collaborativa in un clima scolastico produttivo teso all'orientamento e al miglioramento dell'intero processo di insegnamento apprendimento con un focus sulla centralità dello studente visto come individuo all'interno della scuola.

\section{Riferimenti bibliografici}

Bandura A. (1996): Autoefficacia: teoria e applicazioni. Trad. it. Trento: Erickson, 2000.

Boffo V. (2011): Relazioni educative: tra comunicazione e cura. Milano: Apogeo. Bollnow O.F. (1956): Le tonalità emotive. Trad. it. Milano: Vita e Pensiero, 2008. Bronfenbrenner U. (1979): Ecologia dello sviluppo umano. Trad. it. Bologna: il Mulino, 1986.

Buber M. (1923): Io e Tu. In Id.: Il principio dialogico e altri saggi. Trad. it. Cinisello Balsamo (Mi): Edizioni San Paolo, 1993, pp. 5-66.

Clarizia L. (2017): Lo specifico pedagogico nella professionalità docente. Roma: Edizioni Studium.

Coleman P., Collinge J., Seifert T. (1993): Seeking the Levers of Change: Participant Attitudes and School Improvement. In School Effectiveness and School Improvement, 4(1), pp. 59-83. 
De Beni R., Moé A. (2000): Motivazione e apprendimento. Bologna: il Mulino.

Dewey J. (1916), Democrazia e educazione. Trad. it. Scandicci (Fi): La Nuova Italia, 2004.

Ducci E. (1983): La parola nell'uomo. Spunti per una filosofia dell'educazione. Brescia: La Scuola.

Dusi P. (2012): La comunicazione docenti-genitori: riflessioni e strumenti per tessere alleanze educative. Milano: FrancoAngeli.

Epstein J.L. (2009): School, Family, and Community Partnerships. Your Handbook for Action. Thousand Oaks (CA): Corwin Press.

Eurydice (2008): Levels of Autonomy and Responsabilities of Teachers in Europe. Brussels: Eurydice.

Eurydice (2016): The Teaching Profession in Europe: Practices, Perceptions, and Policies. Brussels: Eurydice.

Eurydice (2020a): Equity in School Education in Europe: Structures, Policies and Student Performance. Brussels: Eurydice.

Eurydice (2020b): The European Higher Education Area in 2020. Bologna Process Implementation Report. Brussels-Luxembourg: Eurydice.

Fabbri L., Striano M., Melacarne C. (2008): L'insegnante riflessivo: coltivazione e trasformazione delle pratiche professionali. Milano: FrancoAngeli.

Goleman D. (1995): Intelligenza emotiva. Trad. it. Milano: Rizzoli, 1997.

Giorgi A., Lo Verso G. (2008): Mente-corpo-relazione: l'unitarietà del vivente. Rivista di Psicologia Clinica, n. 3, p. 285.

Invernizzi E. (2000): La comunicazione organizzativa: teorie, modelli e metodi. Milano: Giuffrè Editore.

Mari G. (2009): La relazione educativa. Brescia: La Scuola.

Mariani L. (2006): La motivazione a scuola. Prospettive teoriche e interventi strategici. Roma: Carocci.

McLuhan M. (1989): The Global Village. Oxford: Oxford University Press.

Mortari L. (2009). Ricercare e riflettere. La formazione del docente professionista. Roma: Carocci.

OECD (2018): Programme for International Student Assessment. Paris: OECD Publishing.

Paletta A. (2013): Valutazione dell' istruzione e miglioramento: il contributo degli studi di management. Torino: Fondazione Agnelli.

Recalcati M. (2011): Cosa resta del padre? La paternità nell'epoca ipermoderna. Milano: Raffaello Cortina.

Rizzolatti G., Sinigaglia C. (2006): So quel che fai. I neuroni specchio e il cervello che agisce. Milano: Raffaello Cortina.

Rogers C. (1957): La terapia-centrata-sul-cliente. Teoria e ricerca. Trad. it. Firenze: Martinelli, 1970.

Seligman M.E. (1990): Imparare l'ottimismo. Come cambiare vita cambiando il pensiero. Firenze: Giunti,1996.

Striano M. (2001): La "razionalità riflessiva" nell'agire educativo. Napoli: Liguori. 
Trotman M.F. (2001, Autumn): Involving the African American Parent: Recommendation to Increase the Level of Parent Involvement within African American Families. The Journal of Negro Education - Special Number, 70(4), pp. $275-285$.

Turnaturi G. (2000). Tradimenti. L'imprevedibilità nelle relazioni umane. Milano: Feltrinelli.

Vygotskij L.S. (1930): Il processo cognitivo. Trad. it. Torino: Bollati Boringhieri, 1987.

Ebner F. (1921). La parola e le realtà spirituali. Frammenti pneumatologici. Trad. it. Cinisello Balsamo (Mi): Edizioni San Paolo, 1998.

\section{Riferimenti normativi nazionali}

DECRETO LEGISLATIVO 13 aprile 2017, n. 66: Norme per la promozione dell'inclusione scolastica degli studenti con disabilità, a norma dell'articolo 1, commi 180 e 181, lettera c), della egge 13 luglio 2015, n. 107 (17G00074) (GU Serie Generale n. 112 del 16-05-2017 - Suppl. Ordinario n. 23).

DECRETO DEL PRESIDENTE DELLA REPUBBLICA 24 giugno 1998, n. 249: Regolamento recante lo statuto delle studentesse e degli studenti della scuola secondaria (GU Serie Generale n. 175 del 29-07-1998).

DECRETO DEL PRESIDENTE DELLA REPUBBLICA 21 novembre 2007, n. 235: Regolamento recante modifiche ed integrazioni al decreto del Presidente della Repubblica 24 giugno 1998, n. 249, concernente lo statuto delle studentesse e degli studenti della scuola secondaria (GU Serie Generale n. 293 del 18-12-2007).

DECRETO DEL PRESIDENTE DELLA REPUBBLICA 16 novembre 2012, n. 254: Regolamento recante indicazioni nazionali per il curricolo della scuola dell'infanzia e del primo ciclo d'istruzione, a norma dell'articolo 1, comma 4, del decreto del Presidente della Repubblica 20 marzo 2009, n. 89 (13G00034) (GU Serie Generale n. 30 del 05-02-2013).

DECRETO DEL PRESIDENTE DELLA REPUBBLICA 16 aprile 2013, n. 62: Regolamento recante codice di comportamento dei dipendenti pubblici, a norma dell'articolo 54 del decreto legislativo 30 marzo 2001, n. 165 (13G00104) (GU Serie Generale n. 129 del 04-06-2013).

LEGGE 7 agosto 1990, n. 241: Nuove norme in materia di procedimento amministrativo e di diritto di accesso ai documenti amministrativi (GU Serie Generale n. 192 del 18-08-1990).

LEGGE 7 giugno 2000, n. 150: Disciplina delle attività di informazione e di comunicazione delle pubbliche amministrazioni (GU Serie Generale n. 136 del 13-06-2000).

LEGGE 13 luglio 2015, n. 107: Riforma del sistema nazionale di istruzione e formazione e delega per il riordino delle disposizioni legislative vigenti (15G00122) (GU Serie Generale n. 162 del 15-07-2015). 
LEGGE COSTITUZIONALE 18 ottobre 2001, n. 3: Modifiche al Titolo V della parte seconda della Costituzione (GU Serie Generale n. 248 del 24-10-2001). MINISTERO DELL'ISTRUZIONE DELL'UNIVERSITÀ E DELLA RICERCA - DIPARTIMENTO PER L'ISTRUZIONE - DIREZIONE GENERALE PER LO STUDENTE, L'INTEGRAZIONE, LA PARTECIPAZIONE E LA COMUNICAZIONE: Nota 31 luglio 2008. Prot. n. 3602/P0. Oggetto: D.P.R. n. 235 del 21 novembre 2007 - Regolamento recante modifiche ed integrazioni al D.P.R. 24 giugno 1998, n. 249, concernente lo Statuto delle studentesse e degli studenti della scuola secondaria.

MINISTERO DELL'ISTRUZIONE DELL'UNIVERSITÀ E DELLA RICERCA: Nota MIUR 22.11.2012. Prot. n. 3214. Trasmissione Linee di indirizzo "Partecipazione dei genitori e corresponsabilità educativa".

MINISTERO DELLISTRUZIONE DELL'UNIVERSITÀ E DELLA RICERCA: Linee di indirizzo per favorire il diritto allo studio degli alunni adottati, e relativa Nota ministeriale, Prot. N. 7443. 